\title{
Topical vancomycin for neurosurgery wound prophylaxis: an interim report of a randomized clinical trial on drug safety in a diverse neurosurgical population
}

\author{
Ryan E. Radwanski, BS, ${ }^{1,2}$ Brandon R. Christophe, BA, ${ }^{1}$ Josephine U. Pucci, BA, \\ Moises A. Martinez, BA, ${ }^{1}$ Michael Rothbaum, BA, ${ }^{2}$ Emilia Bagiella, PhD, ${ }^{3}$ \\ Franklin D. Lowy, MD, ${ }^{1}$ Jared Knopman, MD, ${ }^{2}$ and E. Sander Connolly Jr., MD'
}

\begin{abstract}
${ }^{1}$ Department of Neurological Surgery, Columbia University Medical Center; ${ }^{2}$ Department of Neurological Surgery, Weill Cornell Medical Center; and ${ }^{3}$ Center of Biostatistics, Department of Population Health Science and Policy, The Icahn School of Medicine at Mount Sinai, New York, New York
\end{abstract}

\begin{abstract}
OBJECTIVE Postoperative surgical site infections (SSIs) in neurosurgical patients carry a significant risk of increased morbidity and mortality. With SSIs accounting for approximately $20 \%$ of nosocomial infections and costing approximately $\$ 1.6$ billion USD annually, there is a need for additional prophylaxis to improve current standards of care. Topical vancomycin is increasingly utilized in instrumented spinal and cardiothoracic procedures, where it has been shown to reduce the risk of SSIs. A randomized controlled trial assessing its efficacy in the general neurosurgical population is currently underway. Here, the authors report their initial impressions of topical vancomycin safety among patients enrolled during the 1 st year of the trial.
\end{abstract}

METHODS This prospective, multicenter, patient-blinded, randomized controlled trial will enroll 2632 patients over 5 years. Here, the authors report the incidence of adverse events, the degree of systemic vancomycin absorption in treated patients, and pattern changes of antibiotic-resistant profiles of Staphylococcus aureus flora among patients enrolled during the 1 st year.

RESULTS The topical vancomycin treatment group comprised 257 patients (514 total enrolled patients), of whom 2 exhibited weakly positive serum levels of vancomycin (>3.0 mg/dl). S. aureus was detected preoperatively in the anterior nares of $35(18.1 \%)$ patients and the skin near the surgical site of $9(4.7 \%)$. Colonization in the nares remained for many patients $(71.4 \%)$ through postoperative day 30 . The authors found a significant association between preoperative $S$. aureus colonization and postoperative colonization. Seven methicillin-resistant isolates were detected among 6 different patients. Two isolates were detected preoperatively, and 5 were de novo postoperative colonization. No adverse responses to treatment have been reported to date.

CONCLUSIONS The authors' data indicate that the use of topical vancomycin is safe with no significant adverse effects and minimal systemic absorption, and no development of vancomycin-resistant microorganisms.

Clinical trial registration no.: NCT02284126 (clinicaltrials.gov)

https://thejns.org/doi/abs/10.3171/2018.6.JNS172500

KEYWORDS topical vancomycin; neurosurgery; surgical site infection; antibiotics; wound prophylaxis

$\mathrm{S}$ URGICAL complications account for a significant portion of healthcare-related morbidity, ${ }^{28}$ which can greatly inhibit clinicians' efforts to ameliorate patient disease. Nosocomial infections of the surgical wound, or surgical site infections (SSIs), account for the largest portion of operative complications: approximately $13.6 \%$ of all US hospital complications ${ }^{28}$ and approximately $20 \%$ of all healthcare-associated infections in US hospitals. ${ }^{26}$ Costs have been estimated at $\$ 1.6$ billion USD annually. ${ }^{15}$

Topical application of vancomycin in the operating room is a longstanding practice; however, evidence supporting the antibiotic's efficacy in reducing SSIs has only been reported in the last decade. Currently, topical application of vancomycin is commonly used for instrumented

ABBREVIATIONS AE = adverse event; CUMC = Columbia University Medical Center; LOS = length of stay; MRSA = methicillin-resistant Staphylococcus aureus; SAE = serious adverse event; $\mathrm{SSI}=$ surgical site infection; $\mathrm{WCMC}=$ Weill Cornell Medical Center.

SUBMITTED October 11, 2017. ACCEPTED June 12, 2018.

INCLUDE WHEN CITING Published online December 14, 2018; DOI: 10.3171/2018.6.JNS172500. 
spinal procedures, where it has been shown to reduce the incidence of SSIs from $4.7 \%$ to $0.7 \%$. $^{7,31,32,40,41}$ A similar benefit was demonstrated in cardiothoracic surgery when vancomycin paste was applied to the edges of the sternum during sternotomy closure. ${ }^{5,42}$ Evidence suggests that vancomycin applied topically might also decrease SSIs among neurosurgery patients receiving standard perioperative care (intravenous cefazolin 1 hour prior to incision). Preoperatively, systemic vancomycin is often used for patients with allergy to cephalosporin or penicillin. Even so, intravenous vancomycin has not demonstrated such benefits over intravenous cephalosporin alone. ${ }^{18}$ Intravenous prophylactic antibiotics have variable delivery to the surgical site, which limits their ability to achieve bactericidal concentrations locally with the added risk of adverse systemic effects, including hypersensitivity reactions and renal toxicity. Thus, topical antibiotics may achieve greater local concentration at the surgical site while minimizing systemic toxicity. ${ }^{43}$

Limited research directly addresses the potential risks of vancomycin among neurosurgical patients when applied topically. 1,2,30 True allergic reactions to vancomycin are rare and, to our knowledge, no incidence has been reported following topical use. Promotion of antibiotic resistance is of concern for any antimicrobial agent. When applied topically, we believe that the systemic absorption of vancomycin is low. It stands to reason that its effect on the microbiome is minimal and the risk of developing vancomycin-resistant organisms is low. Systemic absorption of topical vancomycin and microbiology profiles of treated patients have not been previously reported.

To date, there has been no report of a prospective, randomized clinical trial assessing the safety or efficacy of topical vancomycin among a neurosurgical population undergoing craniotomy or noninstrumented spinal procedures. Topical Vancomycin for Neurosurgery Wound Prophylaxis is a randomized controlled trial designed to determine whether prophylactic, topical vancomycin applied directly at the site of craniotomy and noninstrumented spinal procedures reduces the incidence of SSI at postoperative day 30 . This study is ongoing, and a complete report on the efficacy of topical vancomycin in the neurosurgery setting is forthcoming. In an effort to illustrate whether or not topical application of vancomycin is safe for neurosurgery patients, we report adverse events, drug kinetics, and microbiology profiles from the 1st year of enrollment.

\section{Methods}

The Topical Vancomycin for Neurosurgery Wound Prophylaxis study is a prospective, multicenter, randomized, patient-blinded, 2-armed clinical trial (registration no. NCT02284126 [clinicaltrials.gov]). Initial contact and patient recruitment occurs through the outpatient neurosurgical clinics at NewYork-Presbyterian Columbia University Medical Center (CUMC) and NewYork-Presbyterian Weill Cornell Medical Center (WCMC). Outpatients are instructed to use an antibacterial soap, and inpatients are scrubbed below the neck with chlorhexidine preoperatively. To achieve $90 \%$ power with a 2 -sided test at the 0.05 level, 1316 patients per group (2632 total) are expected to be enrolled over a 5-year period. This interim report includes patients enrolled between October 20, 2014, and October 19, 2015. Enrollment is expected to be ongoing through 2019. The study is funded, beginning in February 2014, by the Agency for Healthcare Research and Quality (AHRQ), which is part of the US Department of Health and Human Services.

Patients older than 18 years undergoing craniotomy with planned complete dural or dural-substitute closure or a noninstrumented spinal procedure are screened, provide consent, and are randomized in a 1-to-1 ratio through the Research Electronic Data Capture (REDCap), a secure, Web-based data management system. ${ }^{22}$ Patients are randomized to receive either standard prophylaxis or topical vancomycin at the surgical site in addition to standard prophylaxis. Standard prophylaxis includes intravenous cefazolin 1 hour prior to incision. Attending surgeons may use intravenous vancomycin as standard prophylaxis for patients randomized to the control arm with a cephalosporin or penicillin allergy (positive control group). Otherwise, patients in the control arm receive no vancomycin, topical or intravenous.

Patients undergoing instrumented spinal procedures are excluded from the trial because topical vancomycin is already indicated for this population.,31,32,40,41 In addition, patients are excluded if they have evidence of renal insufficiency, allergy to vancomycin including dermatological manifestations, dizziness, or subjective hearing loss (see Table 1 for a full list of inclusion and exclusion criteria).

\section{Adverse Event Reporting}

Phone interviews were conducted between 14 and 30 days after surgery to assess infections, poor recovery concomitant with wound healing, and adverse events (AEs).

Using a comprehensive checklist, patients were ascertained for signs and symptoms of infection, including fe-

\section{TABLE 1. Summary of study enrollment criteria}

\begin{tabular}{l}
\hline Inclusion criteria \\
\hline Age $>18$ yrs \\
\hline $\begin{array}{c}\text { Patient undergoing craniotomy, craniectomy, or noninstrumented } \\
\text { spinal procedures }\end{array}$ \\
\hline Exclusion criteria \\
\hline Admission creatinine $>1.5$ mg/dl or documented renal insufficiency \\
\hline Allergy to vancomycin, including rashes, dizziness, \&/or subjective \\
hearing loss \\
\hline Preexisting infection at or adjacent to operative site \\
\hline $\begin{array}{l}\text { Planned neurosurgical procedure for vestibular schwannoma, } \\
\text { carotid endarterectomy, transsphenoidal approach }\end{array}$ \\
Cannot obtain informed consent from subject, representative, or \\
surrogate \\
Lack of clinical equipoise for use of vancomycin, as determined by \\
operating surgeon \\
\hline Possible same-day discharge following surgical procedure \\
\hline Instrumented spinal surgery for which vancomycin powder is cur- \\
rently used as standard of care
\end{tabular}


ver or chills, increased pain, redness, swelling or warmth around the surgical site, and drainage fluid, discharge, or pus from the incision. In addition, patients were screened for AEs, including hearing loss and local or systemic allergic reactions. All AEs were reported to the Data and Safety Monitoring Board (DSMB) within 24 hours and collectively reviewed and adjudicated among the DSMB members and principal investigators each quarter.

\section{Serum Analysis}

Systemic absorption of topical vancomycin was monitored using serum vancomycin levels drawn at 6 and 20 hours after wound closure, as vancomycin levels have been shown to peak within the first 24 hours postoperatively. ${ }^{41}$ Serum levels of vancomycin were measured in all study patients who received intravenous or topical vancomycin. An undetectable vancomycin level was defined by the central laboratory as $\leq 3.0 \mathrm{mg} / \mathrm{dl}$ (CUMC) or $\leq 3.5 \mathrm{mg}$ / $\mathrm{dl}$ (WCMC).

\section{Microbiology Analyses}

Microbe cultures were obtained from the anterior nares and surgical site preoperatively before draping occurred, within 48 hours of wound closure, and again at 2 weeks and 3 months following surgery with premoistened culturette rayon-tipped swabs (Becton Dickinson). Patient mailers were used to obtain 2-week and 3-month swabs remotely. Cultures were initially stored at $4^{\circ} \mathrm{C}$ and later were incubated in $6 \%$ sodium chloride-supplemented tryptic soy broth at $37^{\circ} \mathrm{C}$ overnight for supported S. aureus growth. The samples were then plated on mannitol salt agar (Becton Dickinson), which selected for Staphylococcal species ${ }^{20,37}$ for 48 hours at $35^{\circ} \mathrm{C}$. Colonies of $S$. aureus that altered mannitol agar from pink to yellow were then streaked onto sheep blood agar and incubated for 24 hours. The presence of $S$. aureus was then fully ascertained using the Murex StaphAurex rapid latex agglutination test (Remel).

Vancomycin susceptibility testing was completed using Etest, and DNA sequencing was utilized to identify known genes of resistance (i.e., the mec gene complex that is responsible for methicillin resistance $\left.{ }^{3}\right)$. Genotyping of the variable-repeat region of protein A (spa) gene was used to determine clonal lineage of $S$. aureus, and spa types were assigned using StaphType software (version 2.2.1, Ridom).

\section{Statistical Analyses}

Standard univariate analyses were performed using Pearson's chi-square test for categorical variables and the Student t-test for continuous variables, as appropriate. Odds ratios with $95 \%$ confidence intervals were computed for the outcomes of interest. Frequency of serious adverse event (SAEs), AEs, and hearing changes were assessed between treatment groups.

Serum vancomycin was classified as either detectable or nondetectable. Frequency of detectable serum vancomycin was assessed between patients who received topical vancomycin without intravenous vancomycin and those who received intravenous vancomycin alone (positive control) using standard contingency table comparison. The means and standard deviations were calculated for serum vancomycin concentration among those with detectable values and compared positive control patients who received intravenous vancomycin using the Student t-test. Additionally, a Passing-Bablok regression was performed to determine statistical agreement of "detectable" and "nondetectable" values between hospitals.

Microbiology cultures were evaluated based on frequency of pre- and postoperative $S$. aureus colonization of the anterior nares and skin. The treatment and control groups were compared. Differences in colonization according to race/ethnicity (classified as Caucasian, Hispanic, Asian, and African American, among others) were compared using standard contingency table analysis. The mean length of stay (LOS) and BMI were compared between those with and without pre- and postoperative colonization using the Student t-test. Frequency of methicillinresistant $S$. aureus (MRSA) colonization was compared between treatment groups by contingency table analysis.

\section{Results}

\section{Patient Population}

Patient enrollment began October 21,2014, at Columbia University Medical Center (CUMC) and January 7, 2015, at Weill Cornell Medical Center (WCMC). During the 1st year, 514 patients were enrolled, including 257 patients in each of the treatment and control groups. Two hundred eighty of $514(54.5 \%)$ patients were female, and the mean age was $55 \pm 16.87$ years. One patient withdrew from the study for unknown reasons, and 4 cases were rescheduled or canceled after randomization. See Table 2 for a full description of patient demographics.

\section{Adverse Events}

Seven of 514 patients experienced an SAE. SAEs were reported in 2 of $257(0.8 \%)$ patients in the treatment group and 5 of 257 (1.9\%) patients in the control group (OR 2.53, 95\% CI $0.54-12.8 ; \mathrm{p}=0.45)$. Overall, 114 of $514(22.2 \%)$ patients experienced AEs. AEs were reported in 60 of $257(23.3 \%)$ patients in the treatment group and 54 of 257 $(21.0 \%)$ patients in the control group. We found no difference in the frequency and type of AEs between the 2 groups (OR $0.87,95 \%$ CI $0.58-1.31 ; \mathrm{p}=0.60)$. Table 3 provides a summary of $\mathrm{AE}$ occurrences.

Due to uncertainty in the literature regarding the ototoxicity of vancomycin, we regularly assessed for postoperative hearing changes. Twenty-four of 209 (11.4\%) patients in the treatment group and 15 of 212 (7.1\%) patients in the control group reported hearing changes (OR 1.62, $95 \%$ CI $0.82-3.10 ; \mathrm{p}=0.18)$. The remaining patients were lost to hearing follow-up (48 [18.7\%] in the treatment group and $45[17.5 \%]$ in the control group).

\section{Serum Analysis}

Serum vancomycin levels within 24 hours after surgery were recorded in 123 of 514 patients (114 of 257 [44\%] patients from the treatment group and 9 of 257 [3.5\%] patients from the control group). Serum vancomycin levels were positive in 2 of $101(2 \%)$ treatment patients who did not receive intravenous vancomycin prior to surgery and 
TABLE 2. Study demographic summary

\begin{tabular}{lc}
\hline \multicolumn{1}{c}{ Characteristic } & Value \\
\hline No. of patients & $514(100)$ \\
\hline Study site CUMC & $362(70.4)$ \\
\hline Randomized to treatment & $257(50.0)$ \\
\hline Female sex & $280(54.5)$ \\
\hline Race/ethnicity & $41(8.0)$ \\
\hline African American (black) & $22(4.3)$ \\
\hline Asian & $335(65.2)$ \\
\hline Caucasian (white) & $58(11.3)$ \\
\hline Hispanic (Latino/a) & $7(1.4)$ \\
\hline Middle Eastern & $15(2.9)$ \\
\hline Mixed or other & $1(0.2)$ \\
\hline Native American & $1(0.2)$ \\
\hline Pacific Islander & $34(6.6)$ \\
\hline Unknown & $55.09(16.87)$ \\
\hline Mean age, yrs & $27.48(8.51)$ \\
\hline Mean BMI, kg/m²
\end{tabular}

Values are presented as the number of patients (\%) unless stated otherwise. Mean values are presented as the mean (SD).

12 of $13(92 \%)$ treatment patients who received intravenous vancomycin prior to surgery $\left(\chi^{2}=6.62, p<0.001\right)$. Among the 9 patients from the control group, serum vancomycin levels were positive in 0 of $3(0 \%)$ patients who did not receive intravenous vancomycin prior to surgery and 6 of $6(100 \%)$ patients who received intravenous vancomycin prior to surgery $\left(\chi^{2}=1.58, \mathrm{p}=0.114\right)$. The mean positive serum vancomycin level in those who received topical vancomycin with no intravenous vancomycin was $6.3 \pm 1.8 \mu \mathrm{g} / \mathrm{ml}$ compared with $10.7 \pm 3.1 \mu \mathrm{g} / \mathrm{ml}$ in the control group $(\mathrm{p}=0.08)$. A Passing-Bablok regression showed very good agreement between serum vancomycin assays performed at the clinical specimen laboratories of CUMC and WCMC $\left(\mathrm{R}^{2}=0.9868\right)$.

\section{Microbiology Analyses}

We collected preoperative (on the surgery day) and postoperative microbiology cultures from the anterior nares, collected on postoperative days 1-2 in 193 (37.5\%) and the skin in $270(52.5 \%)$ of the 514 patients enrolled. Preoperative colonization of $S$. aureus was observed in the anterior nares for 35 of 193 (1.8\%) and skin for 9 of 270 (3.3\%) patients. Preoperative colonization of the nares was observed in 16 of $99(16.2 \%)$ and 19 of $94(20.2 \%)$ patients in the treatment and control groups, respectively (OR 0.76, 95\% CI $0.36-1.54 ; \mathrm{p}=0.58)$. Preoperative colonization of the skin was observed in 5 of $142(3.5 \%)$ and 4 of $128(2.3 \%)$ patients in the treatment and control groups, respectively (OR 1.13, 95\% CI 0.30-4.31; $\mathrm{p}=0.85$ ).

Postoperative colonization of $S$. aureus was observed in the anterior nares for 37 of 193 (19.2\%) patients. Postoperative colonization of $S$. aureus was observed on the skin for 26 of $270(9.6 \%)$ patients. Postoperative colonization of the nares was observed in 18 of $99(18.1 \%)$ and 19 of 94 (20.2\%) patients in the treatment and control groups, re-
TABLE 3. Summary of adverse events $(n=162)$

\begin{tabular}{lccc}
\hline \multirow{2}{*}{ AE } & \multicolumn{2}{c}{ No. of AEs (\%) } & \begin{tabular}{c} 
Probability of No \\
Association \\
\cline { 2 - 3 } w/ Randomization
\end{tabular} \\
\hline Fever & $5(3.1)$ & $5(3.1)$ & 1 \\
\hline Gastrointestinal & $14(8.6)$ & $7(4.3)$ & 0.1 \\
\hline Pulmonary & $2(1.2)$ & $2(1.2)$ & 1 \\
\hline Urinary tract infection & $11(6.8)$ & $8(4.9)$ & 0.48 \\
\hline Allergy or dizziness & $20(12.3)$ & $22(13.6)$ & 0.75 \\
\hline Edema & $3(1.9)$ & $3(1.9)$ & 1 \\
\hline Wound care & $4(2.5)$ & $7(4.3)$ & 0.36 \\
\hline Other & $19(11.7)$ & $30(18.5)$ & 0.1 \\
\hline
\end{tabular}

spectively (OR 0.88, 95\% CI 0.44-1.75; $\mathrm{p}=0.85)$. Postoperative colonization of the skin was observed in 11 of 142 (7.7\%) and 15 of $128(11.7 \%)$ patients in the treatment and control groups, respectively (OR $0.63,95 \%$ CI $0.29-1.37$; $\mathrm{p}=0.31$ ).

Among the 35 patients with preoperative colonization in the nares, postoperative $S$. aureus colonization of the skin was observed in $6(17.1 \%$; 2 treatment vs 4 control) patients. Postoperative $S$. aureus colonization of both the anterior nares and skin was observed in 3 of 35 (1.6\%; 0 treatment vs 3 control) patients preoperatively colonized in the nares. From the 9 patients preoperatively colonized on the skin with $S$.aureus, 1 (0 treatment vs 1 control) patient remained colonized after surgery.

Preoperative $S$. aureus colonization of the anterior nares was significantly associated with postoperative colonization of the nares (OR 16.01, 95\% CI 6.84-35.3; p < 0.001 ). However, preoperative colonization of the skin near the surgical site was not associated with postoperative colonization of the skin (OR 1.18, 95\% CI 0.10-8.47; p = 0.88 ). Additionally, preoperative colonization of the nares was not associated with postoperative colonization of the skin (Table 4).

There was no observed difference between the treatment and control groups in the likelihood of persistence of preoperative colonization of the nares (OR 1.27, 95\% CI $0.28-5.76 ; \mathrm{p}=0.99$ ) or skin (OR 1.67, 95\% CI 0.07-37.27; $\mathrm{p}=0.99$ ). Similarly, there was no observed difference between the treatment and control groups in the likelihood of de novo postoperative colonization of the nares (OR 0.78, 95\% CI 0.27-2.37; $\mathrm{p}=0.77$ ) or skin (OR 1.46, 95\% CI $0.65-3.17 ; \mathrm{p}=0.41$ ).

There was no difference observed in postoperative colonization rates based on elective or inpatient status. Postoperative $S$. aureus colonization of the nares was observed in 24 of $132(18.2 \%)$ elective cases and 13 of 61 (21.3\%) inpatient cases (OR 0.82, 95\% CI 0.39-1.75; $\mathrm{p}=0.61$ ). Postoperative $S$. aureus colonization of the skin was observed in 19 of $183(10.4 \%)$ elective cases and 7 of 87 (8.0\%) inpatient cases (OR 1.32, 95\% CI 0.53-3.28; $\mathrm{p}=0.54$ ).

There was no significant difference observed in the comparison of preoperative colonization of the nares $\left(\chi^{2}\right.$ $=6.54 ; \mathrm{p}=0.140)$ or skin $\left(\chi^{2}=3.25 ; \mathrm{p}=0.517\right)$ when categorized by race/ethnicity. On subgroup analysis, however, 
TABLE 4. Preoperative and postoperative cultures from the anterior nares and skin in 463 patients

\begin{tabular}{|c|c|c|c|c|c|c|c|c|}
\hline & \multicolumn{2}{|c|}{ Preop Nares Colonization } & \multicolumn{2}{|c|}{ No Preop Nares Colonization } & \multicolumn{2}{|c|}{ Preop Skin Colonization } & \multicolumn{2}{|c|}{ No Preop Skin Colonization } \\
\hline & $\begin{array}{c}\text { Postop } \\
\text { Nares } \\
\text { Colonized }\end{array}$ & $\begin{array}{l}\text { No Postop } \\
\text { Nares } \\
\text { Colonized }\end{array}$ & $\begin{array}{c}\text { Postop } \\
\text { Nares } \\
\text { Colonized }\end{array}$ & $\begin{array}{l}\text { No Postop } \\
\text { Nares } \\
\text { Colonized }\end{array}$ & $\begin{array}{c}\text { Postop } \\
\text { Skin } \\
\text { Colonized }\end{array}$ & $\begin{array}{l}\text { No Postop } \\
\text { Skin } \\
\text { Colonized }\end{array}$ & $\begin{array}{c}\text { Postop } \\
\text { Skin } \\
\text { Colonized }\end{array}$ & $\begin{array}{l}\text { No Postop } \\
\text { Skin } \\
\text { Colonized }\end{array}$ \\
\hline \multicolumn{9}{|l|}{ Randomization } \\
\hline Control & 14 & 5 & 5 & 70 & 1 & 3 & 14 & 110 \\
\hline Treatment & 11 & 5 & 7 & 76 & 0 & 5 & 11 & 126 \\
\hline Total & 25 & 10 & 12 & 146 & 1 & 8 & 25 & 236 \\
\hline \multicolumn{9}{|l|}{ Procedure } \\
\hline Elective/outpatient & 17 & 9 & 7 & 99 & 1 & 6 & 18 & 158 \\
\hline Inpatient & 8 & 1 & 5 & 47 & 0 & 2 & 7 & 78 \\
\hline Total & 25 & 10 & 12 & 146 & 1 & 8 & 25 & 236 \\
\hline \multicolumn{9}{|l|}{ Race } \\
\hline African American (black) & 1 & 0 & 1 & 4 & 0 & 0 & 2 & 12 \\
\hline Asian & 3 & 2 & 2 & 6 & 0 & 0 & 3 & 12 \\
\hline Caucasian (white) & 17 & 8 & 5 & 97 & 1 & 5 & 14 & 147 \\
\hline Hispanic (Latino/a) & 1 & 0 & 2 & 15 & 0 & 1 & 2 & 26 \\
\hline Middle Eastern & 0 & 0 & 0 & 2 & 0 & 0 & 0 & 5 \\
\hline Native American & 0 & 0 & 0 & 0 & 0 & 0 & 0 & 0 \\
\hline Pacific Islander & 0 & 0 & 0 & 1 & 0 & 0 & 0 & 1 \\
\hline Mixed or other & 1 & 0 & 0 & 7 & 0 & 0 & 1 & 9 \\
\hline Declined or unknown & 2 & 0 & 2 & 14 & 0 & 2 & 3 & 24 \\
\hline Total & 25 & 10 & 12 & 146 & 1 & 8 & 25 & 236 \\
\hline
\end{tabular}

we did observe that patients of Asian race were more likely to present with preoperative colonization of the nares than those of non-Asian race, observed in 5 of 13 (38.5\%) Asian patients versus 30 of $180(16.7 \%)$ non-Asian patients (OR $3.13,95 \%$ CI 1.07-9.36; $\mathrm{p}=0.049)$.

No significant difference in LOS or BMI was found based on pre- or postoperative $S$. aureus colonization of the nares or skin compared with those without colonization (Table 5).

Seven culture swabs for 6 different patients contained MRSA strains. Table 6 provides a summary of each sample that was positive for methicillin resistance. We found no differences in rates of MRSA between treatment groups, collection site, or the time of collection. We did not detect vancomycin-resistant strains of $S$. aureus in any of the study patients.

\section{Discussion}

Surgical site infections are a significant cause of mor- bidity, mortality, and cost burden in neurosurgery. $9,11,15,16,19$, 23,39 Our study was designed to determine whether vancomycin powder applied topically to the wound closure further reduces SSIs. Caution has been raised against implementing the widespread prophylactic use of topical vancomycin due to concerns for breeding antibiotic-resistant S. aureus. However, among surgical specialties currently utilizing topical vancomycin antibiotic prophylaxis, this treatment is warranted for operational areas in which SSI carries significant morbidity and mortality concerns. ${ }^{11} \mathrm{~A}$ 2010 report of 2630 consecutive craniotomies for intracranial tumors revealed institutional morbidity and mortality rates due to deep SSI. ${ }^{27}$ Minor additional disabilities were observed in $12.8 \%$ of patients with SSI while major additional disabilities were observed in $7.7 \%$ and death in $5.1 \% .{ }^{27}$ Although these rates are not directly indicative of the entire US neurosurgical SSI population, they indicate that the morbidity and mortality associated with neurosurgical SSIs necessitate the exploration of efficacy and safety of topical vancomycin prophylaxis in neurosurgery.

TABLE 5. LOS and BMI based on S. aureus colonization status

\begin{tabular}{lcccc}
\hline \multicolumn{1}{c}{ Colonization Group } & Mean (SD) LOS, Days & $\mathrm{p}$ Value (vs no colonization) & Mean (SD) BMI, kg/m² & $p$ Value (vs no colonization) \\
\hline Preop colonization of nares & $9.3(28.1)$ & 0.14 & $26.8(5.3)$ & 0.76 \\
\hline Postop colonization of nares & $9.3(27.2)$ & 0.13 & $27.4(4.4)$ & 0.12 \\
\hline Preop colonization of skin & $7.2(3.3)$ & 0.72 & $24.75(2.1)$ & 0.65 \\
\hline Postop colonization of skin & $4.2(3.9)$ & 0.15 & $28.01(6.9)$ & 0.9 \\
\hline
\end{tabular}


TABLE 6. Summary of MRSA-positive cultures

\begin{tabular}{lll}
\hline Collection Site & Study Arm & Time of Collection \\
\hline Anterior nares $^{*}$ & Treatment & $>30$ days postop \\
\hline Incision site $^{*}$ & Treatment & $>30$ days postop \\
\hline Anterior nares & Treatment & $>30$ days postop \\
\hline Incision site & Control & $>30$ days postop \\
\hline Anterior nares & Treatment & Preop \\
\hline Anterior nares & Treatment & Preop \\
\hline Incision site & Control & $<30$ days postop \\
\hline
\end{tabular}

* Same patient.

Data from neurosurgical patients suggest that those identified with MRSA colonization may benefit from specific perioperative care with vancomycin. ${ }^{3}$ Staphylococcus aureus colonizes the anterior nares and skin in more than one-third of hospitalized patients, with $1 \%-5 \%$ colonized by MRSA; colonization at admission is associated with an increased risk of postoperative $S$. aureus infections. ${ }^{14,21}$ Due to the significant number of SSIs resulting from MRSA and methicillin-sensitive strains of $S$. aureus ${ }^{12}$ the previously reported efficacy of topical vancomycin is logical.

Prolonged systemic antibiotic prophylaxis increases the risk of developing resistant organisms in the central nervous system after implantation of medical devices. ${ }^{10}$ Consequently, shorter perioperative courses of antibiotics are recommended. ${ }^{10} \mathrm{~A}$ single topical application of vancomycin is expected to provide needed local prophylaxis for the surgical site while minimizing unnecessary systemic exposure. We observed only 2 patients treated with topical vancomycin who exhibited slightly positive vancomycin serum levels $(6.3 \mu \mathrm{g} / \mathrm{ml})$, compared with those who received intravenous vancomycin $(10.7 \mu \mathrm{g} / \mathrm{ml})$. The difference in serum vancomycin levels was not significantly different; however, the frequency at which systemic absorption was observed suggests that topical vancomycin has minimal systemic effect.

Adverse reactions to intravenous vancomycin include hypotension, phlebitis, chills, skin rash, eosinophilia, neutropenia, flushing of the face and neck, and, in rare cases, drug reaction with eosinophilia and systemic symptoms (DRESS) syndrome, ototoxicity, renal failure, StevensJohnson syndrome, thrombocytopenia, and vasculitis. ${ }^{29}$ Additional gastrointestinal reactions are associated with oral administration of vancomycin..$^{33}$ Limited reports are available regarding topical application; however, previous studies of topical vancomycin in the setting of instrumented spine and cranial procedures have reported no association of vancomycin-related AEs with topical treatment. ${ }^{1,2,4,35,36}$ To our knowledge, this study is the first randomized controlled trial to validate a lack of adverse response to topical vancomycin, specifically no significant postoperative hearing changes.

The most common postoperative infectious organism in cranial surgery is $S$. aureus ${ }^{13}$ of which methicillin-resistant strains confer a great risk for mortality. ${ }^{17}$ Monitoring preand postoperative $S$. aureus colonization provides a means for evaluating potential risk for infection and changes in patients' natural flora. As expected, we found that preoperative $S$. aureus colonization of the anterior nares was significantly associated with postoperative colonization of the same site, while preoperative $S$. aureus colonization of the skin was not significantly associated with postoperative colonization of the same site. This supports that current standards of surgical preparation are, to some degree, effective in eliminating preoperative $S$. aureus colonization near the incision site. However, topical vancomycin does not appear to further reduce the persistence of $S$. aureus skin colonization.

Rates of new colonization of postoperative $S$. aureus may be a risk predictor of infection. Of interest is the subgroup of patients lacking preoperative $S$. aureus colonization of the skin near the surgical site but showing positive S. aureus colonization following surgery (Table 4 column 7, "No Preop Skin Colonization: Postop Skin Colonized"). As expected, topical vancomycin treatment does not significantly increase postoperative $S$. aureus colonization rates of the anterior nares or the skin near the surgical site. It remains unclear whether topical application of vancomycin reduces the risk of $S$. aureus colonization following cranial surgery.

Interestingly, we found that Asian race was associated with a significantly higher rate of preoperative $S$. aureus colonization of the anterior nares. Nasal carriage of $S$. aureus was previously investigated across multiple races/ ethnicities, although no association was found with Asian race; however, the study involved a survey of healthy New Zealand residents. ${ }^{6}$ It is possible that $S$. aureus carriage patterns among the Asian community in New York may vary. Considering the high prevalence of wound infection by $S$. aureus in New York City, the city's Asian population may be at an increased risk, although further data are needed to substantiate this plausibility. ${ }^{34,38}$

Higher BMI and longer LOS both increase the risk of postoperative SSI. ${ }^{8,25}$ However, no association of these risk factors with pre- or postoperative $S$. aureus colonization was found. Based on current data, no evidence exists for selectively using prophylactic, topical vancomycin based on $S$. aureus colonization patterns.

\section{Conclusions}

Our study supports the use of topical vancomycin in cranial and uninstrumented spine neurosurgery. There is minimal systemic absorption, no significant difference in AEs or hearing changes, and no increase in methicillin- or vancomycin-resistant microorganisms. Additionally, topical vancomycin does not appear to significantly change $S$. aureus colonization patterns. We conclude that topical vancomycin treatment is safe in the setting of neurological surgery. For a full description of the study and projected timeline of enrollment, please see the previously published protocol by Jonokuchi et al. ${ }^{24}$

\section{Acknowledgments}

Funding for work was provided by the National Institutes of Health (NIH) through the Agency of Healthcare Research and Quality (AHRQ).

\section{References}

1. Abdullah KG, Attiah MA, Olsen AS, Richardson A, Lucas 
TH: Reducing surgical site infections following craniotomy: examination of the use of topical vancomycin. J Neurosurg 123:1600-1604, 2015

2. Abdullah KG, Chen HI, Lucas TH: Safety of topical vancomycin powder in neurosurgery. Surg Neurol Int 7 (Suppl 39):S919-S926, 2016

3. Akins PT, Belko J, Banerjee A, Guppy K, Herbert D, Slipchenko T, et al: Perioperative management of neurosurgical patients with methicillin-resistant Staphylococcus aureus. J Neurosurg 112:354-361, 2010

4. Alcalá-Cerra G, Paternina-Caicedo AJ, Moscote-Salazar LR, Gutiérrez-Paternina JJ, Niño-Hernández LM: [Application of vancomycin powder into the wound during spine surgery: systematic review and meta-analysis.] Rev Esp Cir Ortop Traumatol 58:182-191, 2014 (Span)

5. Arruda MV, Braile DM, Joaquim MR, Suzuki FA, Alves RH: [The use of the vancomycin paste for sternal hemostasis and mediastinitis prophylaxis.] Rev Bras Cir Cardiovasc 23:35-39, 2008 (Portuguese)

6. Best N, Fraser JD, Rainey PB, Roberts SA, Thomas MG, Ritchie SR: Nasal carriage of Staphylococcus aureus in healthy Aucklanders. N Z Med J 124:31-39, 2011

7. Caroom C, Tullar JM, Benton EG Jr, Jones JR, Chaput CD: Intrawound vancomycin powder reduces surgical site infections in posterior cervical fusion. Spine (Phila Pa 1976) 38:1183-1187, 2013

8. Cassir N, De La Rosa S, Melot A, Touta A, Troude L, Loundou A, et al: Risk factors for surgical site infections after neurosurgery: A focus on the postoperative period. Am J Infect Control 43:1288-1291, 2015

9. Chaichana KL, Bydon M, Santiago-Dieppa DR, Hwang L, McLoughlin G, Sciubba DM, et al: Risk of infection following posterior instrumented lumbar fusion for degenerative spine disease in 817 consecutive cases. J Neurosurg Spine 20:45-52, 2014

10. Chauv S, Fontaine GV, Hoang QP, McKinney CB, Baldwin M, Buckel WR, et al: Risk of resistant organisms and Clostridium difficile with prolonged systemic antibiotic prophylaxis for central nervous system devices. Neurocrit Care 25:128-132, 2016

11. Chiang HY, Kamath AS, Pottinger JM, Greenlee JDW, Howard MA III, Cavanaugh JE, et al: Risk factors and outcomes associated with surgical site infections after craniotomy or craniectomy. J Neurosurg 120:509-521, 2014

12. Chiang HY, Herwaldt LA, Blevins AE, Cho E, Schweizer ML: Effectiveness of local vancomycin powder to decrease surgical site infections: a meta-analysis. Spine J 14:397-407, 2014

13. Dashti SR, Baharvahdat H, Spetzler RF, Sauvageau E, Chang SW, Stiefel MF, et al: Operative intracranial infection following craniotomy. Neurosurg Focus 24(6):E10, 2008

14. Davis KA, Stewart JJ, Crouch HK, Florez CE, Hospenthal DR: Methicillin-resistant Staphylococcus aureus (MRSA) nares colonization at hospital admission and its effect on subsequent MRSA infection. Clin Infect Dis 39:776-782, 2004

15. de Lissovoy G, Fraeman K, Hutchins V, Murphy D, Song D, Vaughn BB: Surgical site infection: incidence and impact on hospital utilization and treatment costs. Am J Infect Control 37:387-397, 2009

16. Edwards JR, Peterson KD, Mu Y, Banerjee S, Allen-Bridson K, Morrell G, et al: National Healthcare Safety Network (NHSN) report: data summary for 2006 through 2008, issued December 2009. Am J Infect Control 37:783-805, 2009

17. Engemann JJ, Carmeli Y, Cosgrove SE, Fowler VG, Bronstein MZ, Trivette SL, et al: Adverse clinical and economic outcomes attributable to methicillin resistance among patients with Staphylococcus aureus surgical site infection. Clin Infect Dis 36:592-598, 2003

18. Finkelstein R, Rabino G, Mashiah T, Bar-El Y, Adler Z, Kertzman V, et al: Vancomycin versus cefazolin prophylaxis for cardiac surgery in the setting of a high prevalence of methicillin-resistant staphylococcal infections. J Thorac Cardiovasc Surg 123:326-332, 2002

19. Gerometta A, Rodriguez Olaverri JC, Bitan F: Infections in spinal instrumentation. Int Orthop 36:457-464, 2012

20. Han Z, Lautenbach E, Fishman N, Nachamkin I: Evaluation of mannitol salt agar, CHROMagar Staph aureus and CHROMagar MRSA for detection of meticillin-resistant Staphylococcus aureus from nasal swab specimens. J Med Microbiol 56:43-46, 2007

21. Harbarth S, Fankhauser C, Schrenzel J, Christenson J, Gervaz P, Bandiera-Clerc C, et al: Universal screening for methicillin-resistant Staphylococcus aureus at hospital admission and nosocomial infection in surgical patients. JAMA 299:1149-1157, 2008

22. Harris PA, Taylor R, Thielke R, Payne J, Gonzalez N, Conde JG: Research electronic data capture (REDCap) - a metadata-driven methodology and workflow process for providing translational research informatics support. J Biomed Inform 42:377-381, 2009

23. Hughey AB, Lesniak MS, Ansari SA, Roth S: What will anesthesiologists be anesthetizing? Trends in neurosurgical procedure usage. Anesth Analg 110:1686-1697, 2010

24. Jonokuchi AJ, Knopman J, Radwanski RE, Martinez MA, Taylor BES, Rothbaum M, et al: Topical vancomycin to reduce surgical-site infections in neurosurgery: Study protocol for a multi-center, randomized controlled trial. Contemp Clin Trials 64:195-200, 2018

25. Karhade AV, Cote DJ, Larsen AM, Smith TR: Neurosurgical infection rates and risk factors: a National Surgical Quality Improvement Program analysis of 132,000 patients, 20062014. World Neurosurg 97:205-212, 2017

26. Klevens RM, Edwards JR, Richards CL Jr, Horan TC, Gaynes RP, Pollock DA, et al: Estimating health care-associated infections and deaths in U.S. hospitals, 2002. Public Health Rep 122:160-166, 2007

27. Lassen B, Helseth E, Rønning P, Scheie D, Johannesen TB, Mæhlen J, et al: Surgical mortality at 30 days and complications leading to recraniotomy in 2630 consecutive craniotomies for intracranial tumors. Neurosurgery 68:1259-1269, 2011

28. Leape LL, Brennan TA, Laird N, Lawthers AG, Localio AR, Barnes BA, et al: The nature of adverse events in hospitalized patients. Results of the Harvard Medical Practice Study II. N Engl J Med 324:377-384, 1991

29. Lexicomp: Vancomycin: drug information. UpToDate. (https://www.uptodate.com/contents/vancomycindrug-information?search=Vancomycin:\%20Drug\%20 Information \&source=search_result $\&$ selectedTitle $=1 \sim 148 \&$ usage_type=default \&display_rank=1) [Accessed August 23, 2018]

30. Mallela AN, Abdullah KG, Brandon C, Richardson AG, Lucas TH: Topical vancomycin reduces surgical-site infections after craniotomy: a prospective, controlled study. Neurosurgery [epub ahead of print], 2017

31. Molinari RW, Khera OA, Molinari WJ III: Prophylactic intraoperative powdered vancomycin and postoperative deep spinal wound infection: 1,512 consecutive surgical cases over a 6-year period. Eur Spine J 21 (Suppl 4):S476-S482, 2012

32. O'Neill KR, Smith JG, Abtahi AM, Archer KR, Spengler DM, McGirt MJ, et al: Reduced surgical site infections in patients undergoing posterior spinal stabilization of traumatic injuries using vancomycin powder. Spine J 11:641-646, 2011

33. Osawa R, Kaka AS: Maculopapular rash induced by oral vancomycin. Clin Infect Dis 47:860-861, 2008

34. Pardos de la Gandara M, Raygoza Garay JA, Mwangi M, Tobin JN, Tsang A, Khalida C, et al: Molecular types of methicillin-resistant Staphylococcus aureus and Methicillinsensitive $\mathrm{S}$. aureus strains causing skin and soft tissue infec- 
tions and nasal colonization, identified in community health centers in New York City. J Clin Microbiol 53:2648-2658, 2015

35. Rasouli JJ, Kopell BH: The adjunctive use of vancomycin powder appears safe and may reduce the incidence of surgical-site infections after deep brain stimulation surgery. World Neurosurg 95:9-13, 2016

36. Ravikumar V, Ho AL, Pendhakar AV, Sussman ES, KwongHon Chow K, Li G: The use of vancomycin powder for surgical prophylaxis following craniotomy. Neurosurgery 80:754-758, 2017

37. Sharp SE, Searcy C: Comparison of mannitol salt agar and blood agar plates for identification and susceptibility testing of Staphylococcus aureus in specimens from cystic fibrosis patients. J Clin Microbiol 44:4545-4546, 2006

38. Shopsin B, Mathema B, Martinez J, Ha E, Campo ML, Fierman A, et al: Prevalence of methicillin-resistant and methicillin-susceptible Staphylococcus aureus in the community. J Infect Dis 182:359-362, 2000

39. Steiner C, Elixhauser A, Schnaier J: The healthcare cost and utilization project: an overview. Eff Clin Pract 5:143-151, 2002

40. Strom RG, Pacione D, Kalhorn SP, Frempong-Boadu AK: Decreased risk of wound infection after posterior cervical fusion with routine local application of vancomycin powder. Spine (Phila Pa 1976) 38:991-994, 2013

41. Sweet FA, Roh M, Sliva C: Intrawound application of vancomycin for prophylaxis in instrumented thoracolumbar fusions: efficacy, drug levels, and patient outcomes. Spine (Phila Pa 1976) 36:2084-2088, 2011

42. Vander Salm TJ, Okike ON, Pasque MK, Pezzella AT, Lew $\mathrm{R}$, Traina V, et al: Reduction of sternal infection by application of topical vancomycin. J Thorac Cardiovasc Surg 98:618-622, 1989

43. Xiong L, Pan Q, Jin G, Xu Y, Hirche C: Topical intrawound application of vancomycin powder in addition to intravenous administration of antibiotics: a meta-analysis on the deep infection after spinal surgeries. Orthop Traumatol Surg Res 100:785-789, 2014

\section{Disclosures}

The authors report no conflict of interest concerning the materials or methods used in this study or the findings specified in this paper.

\section{Author Contributions}

Conception and design: Radwanski, Lowy, Knopman, Connolly. Acquisition of data: Radwanski, Martinez, Rothbaum, Lowy, Knopman, Connolly. Analysis and interpretation of data: Radwanski, Christophe, Connolly. Drafting the article: Radwanski, Christophe, Pucci, Connolly. Critically revising the article: Radwanski, Christophe, Pucci, Bagiella, Connolly. Reviewed submitted version of manuscript: all authors. Approved the final version of the manuscript on behalf of all authors: Radwanski. Statistical analysis: Radwanski, Christophe, Pucci, Bagiella. Administrative/ technical/material support: Radwanski, Christophe, Pucci, Martinez, Rothbaum, Connolly. Study supervision: Martinez, Bagiella, Lowy, Knopman, Connolly.

\section{Supplemental Information}

\section{Previous Presentations}

Portions of this work were presented in abstract form at the 84th American Association of Neurological Surgeons (AANS) Annual Scientific Meeting, Chicago, Illinois, April 30-May 4, 2016.

\section{Correspondence}

Ryan E. Radwanski: Columbia University Medical Center, New York, NY.rer2022@med.cornell.edu. 